\title{
A Hybrid Approach for Selecting a Course Management System: A Case Study
}

\author{
Anil Aggarwal, Veena Adlakha, and Terry Ross \\ University of Baltimore, Baltimore, Maryland, USA
}

\section{aaggarwal@ubalt.edu vadlakha@ubalt.edu tross@ubalt.edu \\ Executive Summary}

The Internet is creating exciting opportunities for universities and their stakeholders. Universities are experimenting with different modes of education. E-learning is one such mode, where students can learn from any place at any time, as long as they have an Internet connection. A course management system (CMS) facilitates e-learning, making it critical to select a system that can meet the needs of all its stakeholders. This paper addresses a hybrid approach used to select a course management system for a mid-Atlantic university. The resulting product is the outcome of faculty input, inputs from the program manager of e-Learning, students, and other stakeholders. The authors have experienced four different CMSs over twelve years of teaching online. Instead of re-inventing the wheel, universities planning to venture into e-learning or trying to revamp their current CMS can learn from our experiences. The case study could assist current and future Web course faculty in selecting a CMS to suit their teaching needs. In addition, by increasing their awareness of various capabilities that might be present in their current CMS, the discussion in the paper could also encourage the faculty to revamp their courses.

Keywords: e-Learning, Course Management System, Case Study, Sakai, Blackboard, Moodle.

\section{Introduction}

As we move from a face-to-face to an Internet-based environment, organizations are preparing roadmaps to take advantage of this change. Businesses, non-profit organizations, governments, and even academia are re-defining their missions to include web-based components (Picciano, Seaman, \& Allen, 2010). This is creating exciting opportunities for all stakeholders to innovate and become part of the digital revolution. Universities are re-inventing themselves by offering a virtual learning environment (VLE). According to Dillenbourg (2000, p. 2), "a virtual learning environment is a designed information space"; because the space is designed, Dillenbourg refers to the "architecture" of information rather than its "structure" or "organization." The software system that facilitates this environment and provides much of its architecture is a Course Management System (CMS). A CMS is an Internet-based software program that provides a set of

Material published as part of this publication, either on-line or in print, is copyrighted by the Informing Science Institute. Permission to make digital or paper copy of part or all of these works for personal or classroom use is granted without fee provided that the copies are not made or distributed for profit or commercial advantage AND that copies 1) bear this notice in full and 2) give the full citation on the first page. It is permissible to abstract these works so long as credit is given. To copy in all other cases or to republish or to post on a server or to redistribute to lists requires specific permission and payment of a fee. Contact Publisher@InformingScience.org to request redistribution permission. integrated tools for assessment and evaluation, content development, content management and delivery, communication, security, and course administration. A CMS enables faculty to go beyond the time and space constraints of traditional face-to-face teaching, allowing them to post information on the web without any knowledge or understanding of technical details of the CMS. 
Christensen (2008, p. 1) and Christensen and Horn (2008, p. 1) in landmark papers address the concept of disruptive innovation and suggest "... the way to implement an innovation is to implement it disruptively. Schools should not use technology to compete against the existing paradigm and serve existing customers, but let it compete against 'non-consumption,' where the alternative is nothing at all." They suggest that (1) educational video games could make learning fun and motivating, and (2) computers offer a way to customize instruction and allow students to learn in the way they are best wired to process information. According to Walter (2010, para. 8), "A disruptive innovation initially offers an alternative option to a previously established method of delivery." Information and communication technology (ICT) has disrupted the traditional way of learning by making learning convenient for all from a perspective of anytime anyplace (Sangrà \& González-Sanmamed, 2011). This change has opened new educational paradigms, motivating universities to offer anytime anyplace learning through Web-Based Education (WBE). There is abundant literature on the importance and diffusion of web-based learning. For example, Aggarwal and Makonnen (2008) discuss factors needed for successful globalized e-learning.

The CMS market is becoming increasingly lucrative as more and more schools and organizations offer web-based courses and training (Sener, 2010). However, not many researchers have discussed the desirable features of a stakeholder-friendly CMS. Adlakha and Aggarwal (2009) provide a list of the minimum functionality needed for a CMS from the faculty perspective. Their study is based on their extensive online teaching experiences with various platforms, as well as interaction with colleagues and academic peers at other institutions. They conclude that institutions entering a VLE or looking to change their current CMS could use the features discussed in the paper to evaluate and compare the CMS proposals under consideration. Unal and Unal (2011) discuss usability of two widely used course management systems, Moodle and Blackboard, from students' perspective and conclude that Moodle is favored by students over Blackboard for most features. The study in our paper, however, goes a step further and discusses CMS functionality from all stakeholders' perspectives.

On the procurement issues, Santala and Samuli (2011) defined Spend Management and Analysis as a way of monitoring procurement costs. Al-Mahmood and Mansoor (2011) defined an integrated approach to COTS software procurement process, and many others have defined standard procurement processes for commercial enterprises. Academia is a special case because of its nonprofit nature and its core mission of education. Standard COTS approaches which may be economically-driven may need to be modified to cater to educational needs. Moreover, academia must use an approach which satisfies its main stakeholders: students and faculty. This necessitates a combination of processes which takes steps from various methodologies. Laudon and Laudon (2010) have described many different approaches, such as the traditional System Development Life Cycle (SDLC), Rapid Application development, Component-Based Development, and Joint Application Development (JAD) for system building. Kerzner (2009) discusses several examples of project management for different applications.

This paper presents the hybrid process used at one university for selecting a course management system (CMS). The University of Baltimore (UB) is one of the first institutions to offer WBE and, as a result, has become a pioneer in offering online education. The university has used three different CMSs over the last ten years and recently selected a fourth system. We refrain from discussing all earlier CMSs used by the university, as the purpose here is to discuss the process that is used in selecting a CMS. The process itself does not follow any single defined methodologies like the system analysis and design (SDLC), project management, commercial off-the-shelf software (COTS) and spend analysis, but is a combination of several approaches. The selection process used a SDLC approach (Laudon \& Laudon, 2010) and combined it with prototyping and project management to create a hybrid approach. Specifically, we took the SDLC and modified it for our selection process and used project management approach for the final CMS selection. 
Once the system was selected, designers used prototyping to refine the system for end users. The next section discusses the case.

\section{A Case Study}

Since launching its WBE program in 1999, our university has sought to continuously improve the CMS software either out of necessity or capability. Due to mergers and acquisitions of CMS software companies and cost constraints, the university has worked on four different platforms. In late 2010, the university was informed that its CMS vendor would no longer provide the current CMS platform. This necessitated the selection of a new CMS system. As already mentioned, no formal system/project approach was used, the various actions undertaken by the university mirrored a typical System Development Life Cycle (SDLC) and project management approach. There are many different versions of SDLC; we used the SDLC steps (Planning, Analysis, Design, Development, Testing, Implementation and Maintenance) recommended by Haag and Cummins (2010) and have modified and customized them to apply to the proposed CMS selection process. Many resources (personnel, office space, etc.) and specifications (window environment, PC type, etc.) were pre-determined and were used as constraints during the selection process; hence we modified SDLC's steps into a hybrid framework applicable to our project as presented in Table 1.

\begin{tabular}{|c|c|}
\hline \multicolumn{2}{|c|}{ Table 1: The hybrid framework } \\
\hline Modified SDLC & Steps in Hybrid Framework \\
\hline Planning & $\begin{array}{l}\text { - } \text { Project scope } \\
\text { - Develop the project plan } \\
\quad \text { o Identify stakeholders \& champions }\end{array}$ \\
\hline Analysis & $\begin{array}{l}\text { - } \quad \text { Gather stakeholder requirements } \\
\text { - } \quad \text { Identify CMS requirements } \\
\text { - } \quad \text { Initiate a Request for Proposals (RFP) } \\
\text { Process and Select }\end{array}$ \\
\hline Testing & Rank/Test system(s) for Functionality \\
\hline $\begin{array}{l}\text { Selection and } \\
\text { Modification }\end{array}$ & $\begin{array}{l}\text { - } \text { Select a system } \\
\text { - } \text { Operationalize the system } \\
\text { - } \quad \text { Modify to meet stakeholder's requirements }\end{array}$ \\
\hline Implementation & $\begin{array}{ll}\text { - } & \text { Train stakeholders } \\
\text { - } & \text { Make it fully operational }\end{array}$ \\
\hline
\end{tabular}

In the following sections we discuss, under the sub-headings as presented in Table 1, the CMS selection process by highlighting some of the challenges we encountered and the steps that were taken to overcome these challenges. 


\section{Planning}

\section{Project Scope}

A CMS facilitates content development, management, and delivery, and it assists in student performance assessment. A CMS is, thus, a critical component of any web-based education. The motivation for the project was based on necessity since the current CMS provider was withdrawing its product and support. In that sense the scope of the project was predefined as select a viable CMS system to replace the existing system. Since the scope was predetermined, it will not be discussed further.

\section{Develop the Project Plan}

\section{Identify stakeholders and champions}

The first critical task was to identify stakeholders, because the success or failure of a CMS depends on stakeholders' satisfaction. Strong, Ringer, and Taylor (2001) discuss critical factors for stakeholder's satisfaction as the timeliness of communication, the honesty and completeness of the information, and the empathy and equity of treatment by management. Babou (2008) in a stakeholder's analysis posting identifies the essential steps for stakeholder analysis as:

- Stakeholder identification and documenting their interests.

- Assessing the power of, importance of \& level of impact upon each stakeholder.

- Identifying how best to engage stakeholders in the project by analyzing their reactions or response in different situations.

Using Babou's (2008) satisfaction criteria, we identified and classified stakeholders as:

- Faculty - they conduct online classes and probably use CMS extensively in web-based or hybrid courses. They have high power: they must be engaged throughout and must be satisfied.

- Students - they take online classes and probably use CMS extensively in web-based or hybrid courses. They have high power: must be engaged throughout and must be satisfied.

- Administrators - they monitor the whole process and ensure economic, technical, and operational feasibilities. They have high power, and their initial engagement is high since they are responsible for starting and moving the project forward. Once the project starts, they facilitate the process and then become less engaged.

- Programmers - They are technical stakeholders who are involved in tweaking the system once it is selected. They have low to medium power, and their initial engagement is limited, but once a CMS is selected their interest becomes high.

- Operators/Help Desk - They typically do not have much power in CMS selection.

Based on Babou's classifications above, we developed Table 2 to provide a generic template for stakeholder analysis in a CMS selection process. This template can be modified for an individual situation. 


\begin{tabular}{|l|l|l|l|}
\hline \multicolumn{5}{|c|}{ Table 2: Stakeholder's Analysis } \\
\hline Stakeholder & $\begin{array}{c}\text { Interest Level (stake } \\
\text { in success) }\end{array}$ & $\begin{array}{c}\text { Power (Influence } \\
\text { over CMS selection) }\end{array}$ & Level of Engagement \\
\hline Faculty & High & High & High \\
\hline Student & High & Medium & High (later) \\
\hline Administrators & Medium & High & $\begin{array}{l}\text { High (initially) } \\
\text { Medium (later) }\end{array}$ \\
\hline Programmers & High & Medium & $\begin{array}{l}\text { Low (initially) } \\
\text { High (later) }\end{array}$ \\
\hline Operators/Help Desk & Medium & Low & Low \\
\hline
\end{tabular}

Once the stakeholders were identified, it was necessary to find "ambassadors" in each stakeholder group. Ambassadors, here, are defined as individuals who are familiar with a CMS system, know the scope of the current project, and have high power and high stake in CMS success. Based on the above definition, a group of faculty, students, and administration were identified as part of a task force whose goal was to assist in CMS selection. A task force was formed consisting of 12 members from the faculty, the Office of Technology Services (OTS), and library staff. Students were indirectly involved in the assessment process once the feasible systems were identified. Individuals were selected based on their familiarity with a CMS, their interest in a new CMS, and/or their job requirements (for example, an office of technology (OTS) person may not have a choice if their job requires them to be responsible for hardware and software selection and procurement).

\section{Analysis}

\section{Gather Stakeholder Requirements}

As we move from an old to a new CMS system, it is natural for stakeholders to desire features that were not available before but also to keep desirable features that were available in the older CMS. Using old and new desirable features a "wish" list was generated. It is important to synthesize the wish list from different stakeholder's perspectives. This list can be generated using different techniques like:

- Questionnaire

- Focus groups

- Brainstorming

Questionnaires can be used to identify desirable features of current CMS, whereas focus groups and brainstorming can be used to develop a list of additional desired features of the new CMS.

Adlakha and Aggarwal (2009) provide a comprehensive list of minimal requirements of a CMS. A questionnaire with a similar set of features was developed. All students and faculty that were teaching web courses were encouraged to participate in an online questionnaire. Broadcast e-mail to all faculty members was also sent. In addition, focus groups from the task force were used to study desirable functionalities of the new system. A full inventory of all online courses was taken in order to determine the extent to which the various components of the current CMS were used. 
A list of features was generated and was divided into three parts: critical features, less critical features, and desirable features. These features were further expanded as discussed next.

\section{Identify CMS Requirements}

The above stakeholders' requirements were translated as CMS requirements. Table 3 provides a mapping of stakeholders and the resulting CMS requirements.

\begin{tabular}{|c|c|c|}
\hline \multicolumn{3}{|c|}{ Table 3: Mapping of Stakeholders and CMS features } \\
\hline Features & Stakeholders & CMS Component \\
\hline Critical & $\begin{array}{l}\text { - } \text { content preparation } \\
\text { - } \text { content delivery } \\
\text { - } \text { content management } \\
\text { - } \text { assessment }\end{array}$ & $\begin{array}{l}\text { - } \text { Announcement } \\
\text { - } \text { Syllabus } \\
\text { - } \text { Course Content } \\
\text { - } \text { WYSIWYG editor } \\
\text { - Conference } \\
\text { - Study groups } \\
\text { - Class members (roster) } \\
\text { - Assignment folders } \\
\text { - Assessment } \\
\text { - Student Portfolio } \\
\text { - Gradebook } \\
\text { - Calendar/Scheduler } \\
\text { - Statistics }\end{array}$ \\
\hline Less Critical & $\begin{array}{ll}\text { - } & \text { synchronous communica- } \\
\text { tion }\end{array}$ & $\begin{array}{ll}\text { - } & \text { Webliography } \\
\text { - } & \text { Workbook } \\
\text { - } & \text { Chat } \\
\text { - } & \text { Class Awareness }\end{array}$ \\
\hline Desirable & $\begin{array}{ll}\text { - } & \text { Triggers } \\
\text { - } & \text { file management } \\
\text { - } & \text { online tutorials } \\
\text { - } & \text { Mobile access } \\
\text { - } & \text { Social networking }\end{array}$ & $\begin{array}{ll}\text { - } & \text { Automatic Notification } \\
\text { - } & \text { Batch Downloading and } \\
\text { Uploading of Assignments } \\
\text { - } & \text { File Management } \\
\text { - } & \text { Virtual Computer Labora- } \\
\text { tory } \\
\text { - }\end{array}$ \\
\hline
\end{tabular}


The above features are described in more detail below with regards to the current CMS system, WebTycho, as the benchmark, while possible enhancements are noted. WebTycho was used as the benchmark because of stakeholders' familiarity with its features.

\section{Critical features}

Announcements: Online courses require frequent announcements, reminders, and notifications. The announcements feature in WebTycho is a single page. Its location makes it impossible to miss (it is the landing page for a course). Desired features in a new CMS's announcements feature might include the ability to create multiple announcements, to open and close them according to a schedule, to archive them, and to copy them from one course to another.

Syllabus: The syllabus may be the single most important component of a Web-course. A CMS could have several generic syllabus templates. The syllabus feature should allow faculty to build a syllabus by filling in items in a fixed template, or by creating alternative (or additional) items, or by pasting an entire syllabus into a single text box. Files may be attached to sections of the syllabus.

Course content: Content preparation can be a challenge in a Web-course. Online courses require a different approach than face-to-face courses. This is where the CMS can provide flexibility in terms of linking sites, integration of publisher e-packs, embedded exercises, and hyperlink navigation. For example, in WebTycho, faculty may create session pages and paste text into them or attach files. Files attached to the "place holder for files" may be linked to from anywhere in the course. It might be desirable to have larger limits on the size of files that may be attached, to allow the uploading of zipped files, and to have a subdirectory or folder structure within the files area.

WYSIWYG ("what you see is what you get") editor: WebTycho's Text Formatting Editor (TFE) may be turned on by the individual user, but the default editor is plain-text. Text created in WORD can be pasted into a TFE textbox and will retain much of its formatting. An instructor can use the TFE to add links to items in the course content area. The TFE includes a spellchecker. It would be desirable if WORD formatting were carried over more fully. Special characters (such as curly quotes) should not cause problems. The WYSIWYG editor should be the default for all users in a new CMS, but one should always be able to work in HTML source.

Conferences: Forum discussion is one of the most commonly used pedagogical techniques in an online classroom. Proper attention to the design, facilitation, and maintenance of an online instructional discussion is critical to promoting students' constructive thinking. This involves twoway communication, where a student can create threads, post responses, and ask questions, and where faculty can create new threads, discuss, or respond to students' questions. Faculty should be able to create multiple conferences ahead of time and be able to open them on desired dates. There should be special symbols (like red dots, a message, or an asterisk) posted besides posts to distinguish those that have been read and those that are unread; it would be useful to be able to view all unread posts in a single large page. Faculty should be able to decide within a course whether students will have the ability to edit their own post after submission. It is convenient to be able to gather all the conference posts for a particular thread (or even for an entire conference) into one large page. Posting and word counts should be available for each student's contributions. It would be desirable if study group conferences could be gathered and counted along with main conference posts. It would also be useful to be able to sort conference posts, threads, and entire conferences by topic, by date, or by author. The ability for Faculty to make comments to a post that would be seen only by the original author and not by other students would help maintain the privacy of students. 
Study groups: Groups may be created for many reasons, such as small group discussions or for group assignments. In a large course, it can be very difficult to keep track of threads to which dozens of students are responding. This can result in a discussion overload, making it difficult for faculty and students to follow threads. Moreover, certain topics may not be worthy of discussion by a large group. To avoid this it is better to have small group discussions. Group/team formation may also be necessary to carry out research or project assignments. Faculty should have the ability to add students to multiple study groups and students should be able to see the content only of groups to which they belong. Groups should have access to a discussion/conference area and a chat room. Faculty members should have access to all study groups.

Class members: A class roster with links to student profiles should be provided at the beginning of the semester. The list should be updated daily to account for adds and drops - the currency of list is very important since it is needed to maintain $24 / 7$ contact with students. It should be possible to send e-mail through a dedicated server instead of user's default email program. This would allow Faculty to maintain contact from any anywhere at any time.

Assignments folder: An assignment submission area independent of other areas is desirable to allow students to submit and for the faculty to view the assignments. In WebTycho, this is where students hand in assignments that are to be viewed only by faculty. As a minimum, from the stakeholder's perspective, the CMS should allow for the following dates:

- Open date: this is the date when assignment is available to students.

- Close date: this is the date students must submit their assignment to get full credit.

- Lock date: no assignment will be accepted after this date. This allows for late submissions with penalty.

- Feedback date: this is the date the faculty feedback is available.

Assessments: A critical part of the learning process is the assessment of student performance during the semester. Student assessment involves all activities that measure student learning and ultimately results in a grade for the course. Multiple assignments modes are desirable, including:

- $\quad$ True/False

- Multiple Choice

- Fill in the Blank

- Matching

- Survey

- Essays

- Multimedia Recordings

WebTycho's assessment tool does a reasonably good job with multiple choice and true-false questions. Assessments may be set up in advance to open and close at specific times. The order of questions on an exam may be scrambled so that no two students have exactly the same test. Scores are automatically sent to the gradebook. Feedback may be withheld while the exam is open and then later released. However, WebTycho does not provide a timestamp when a student submits the exam, which would be beneficial. It would also be useful to get exact reports of how long students spent on exams and the exact time of submission, to be able to offer tests that were divided in sections (with scrambling an option within each section), and to be able to grade essays either by student or by question. For take home exams or online exams that are not multiple- 
choice, it would be desirable to have a time function which can close the exam after the allocated time. It would be useful to have following timings related to assessment:

- Start time

- Finish time

- Date/Time submitted

Student portfolio: In the current CMS, there is a portfolio area that links to all student submissions in assessments, assignments, and open conferences. It would be useful to be able to see the texts of study group conference posts collated with main conference posts. It would also be helpful if portfolios could be downloaded as zipped files.

Gradebook: The Gradebook in a CMS should allow different assignment weights; assignments may be graded on the basis of points, percentages, pass/fail, or letters. The CMS should allow individual grading or group grading of assignments. It would be desirable to be able to import/export a gradebook file into spreadsheet software. In WebTycho, files submitted by students may only be downloaded by Faculty one at a time. The ability to gather all submissions from students into a single zipped file was strongly desired by faculty. Many Faculty allow students to drop their lowest grade or two; the CMS gradebook should allow for that option. A gradebook should provide students with an approximation of their current overall grade at any point during the term.

Calendar/Scheduler: Students tend to forget assignments, exams, and other relevant dates. It would be desirable to have a calendar that lists daily class requirements of readings, discussions, due dates for assignments, quiz/exams, etc. The calendar should emulate popular devices (like the iPhone, Blackberry, etc.) to minimize student learning time, since many students are already using such devices. In addition, it would be highly desirable if the calendar with highlighted due dates could be downloaded to student's portable devices.

Statistics: In online classes, one of the major requirements for students is participation in the class. It is essential to have a robust statistical summary of the following:

- Student visits to the course site

- Time spent each week on
- Discussion
$\circ$ Readings
○ Group

This allows an instructor to monitor student progress. It would be desirable to have automatic filters that provide lists of students who may be falling behind. This filter could create automatic triggers and warn students of their lack of participation.

\section{Less critical features}

Webliography: Both students and faculty may add items to the Webliography in WebTycho. Faculty may edit, reorder, or delete entries. It would be useful to be able to classify entries topics and/or dates.

Workbook: WebTycho has a workbook area that has many features of the assignment folder, except that it is not connected to the gradebook. It is an area where students may prepare drafts of assignments. Faculty cannot see a student's workbook unless the student submits it for review. Faculty may add comments in the workbook, but not grades. 
Chat: The Chat room in a CMS allows real time discussion in public/private groups. It should allow for audio/video interaction, and chat sessions should be saved automatically.

Class awareness: This is the WebTycho's version of instant messaging although it unfortunately identifies people by their usernames (e.g., "UB99X66") rather than by their display names (e.g., "John Smith"). It would be better if display names are used in messaging.

\section{Other desirable features}

Automatic notification: Automatic notifications may be sent to students when there have been changes in the course, or the submission of new conference posts, or for very important announcements. It would be practical if Faculty had the option to turn the notification on and off, and if they could set it to digest mode, rather than having a new email sent every time there is action in the course.

Batch downloading/uploading of assignments for grading: Such a feature could save a considerable amount of time, especially for a large class.

File management features: This could include the ability to upload larger files than WebTycho allows as well as the ability to upload zipped files that will open in the course content area. It would also be useful to be able to create folders or subdirectories within the file area.

Virtual computer laboratory: Virtual laboratories are desirable feature for students in general (Burd, Seazzu, \& Conway, 2009) but especially useful for online students. This would be beneficial for all stakeholders. Students can experiment from home, and Faculty can create webcast lectures to explain working and capabilities of the lab.

Podcasting: In future, it is likely that students may desire lectures and other class related material on their mobile. It would desirable for a CMS to provide this capability.

Social media: The millennium students are spending more time on social media, and it maybe possible to teach students using those resources. It would be desirable to provide these accesses. However, one must be careful not to replace learning with social media chatting.

\section{Initiate an RFP Process and Select Potential CMSs}

Vendors were solicited after discussions with peer schools and other institutions that were using a CMS. The criteria of stability, popularity, operational ease, and technical capability were used for selection of the CMSs for further testing. Based on internet research and vendor presentations, the following three systems were selected:

- Sakai

- Moodle

- Blackboard

Sakai is a community of academic institutions, commercial organizations, and individuals who work together to develop a common Collaboration and Learning Environment (CLE). It is a free educational software platform distributed under the Educational Community License. Moodle is an also open source CMS that has become very popular as a tool for creating online dynamic sites for teaching online provided by Moodle.org. Blackboard is a proprietary system offered by Blackboard.com. 


\section{Testing}

\section{Rank/Test System(s) for Functionality}

To start the ranking procedure, the task force members were given student access to instances of the same course in each of the three systems. They viewed course content, submitted assignments, took exams, and participated in discussion forums. A course site in our current CMS was established for the task force to share their observations about each of the three new systems under examination. In the next phase, a course selected by each faculty member of the task force was made available in each of the three CMSs. This allowed participants to compare the three systems. Students (graduate assistants) were also asked to evaluate the systems. Once members had a chance to evaluate these systems individually, they evaluated them together as a group in real time. This allowed individuals to compare and contrast each other's experiences with each of the three systems. This activity was performed as a structured session with the aim to promote discussion of the pros and cons of each system as perceived by the team members.

A rubric (see Figure 1) was developed to rank and rate each of these systems with the current benchmark, WebTycho. Members were asked:

- To rate each of the three CMSs for each of the above functionalities as compared to the current CMS using a 5-point scale where "1" was "Substantially inferior to WebTycho"; "3" was "Roughly equivalent to WebTycho," and "5" was "Far superior to WebTycho."

- To rank each of the three CMSs on each of the above functionalities on order from one (1) to three (3), where 1 was the top choice.

In addition, each functionality had an area for comments. Figure 1 displays ranking and rating used for the Announcement functionality in the CMS Task Force Survey (2010):

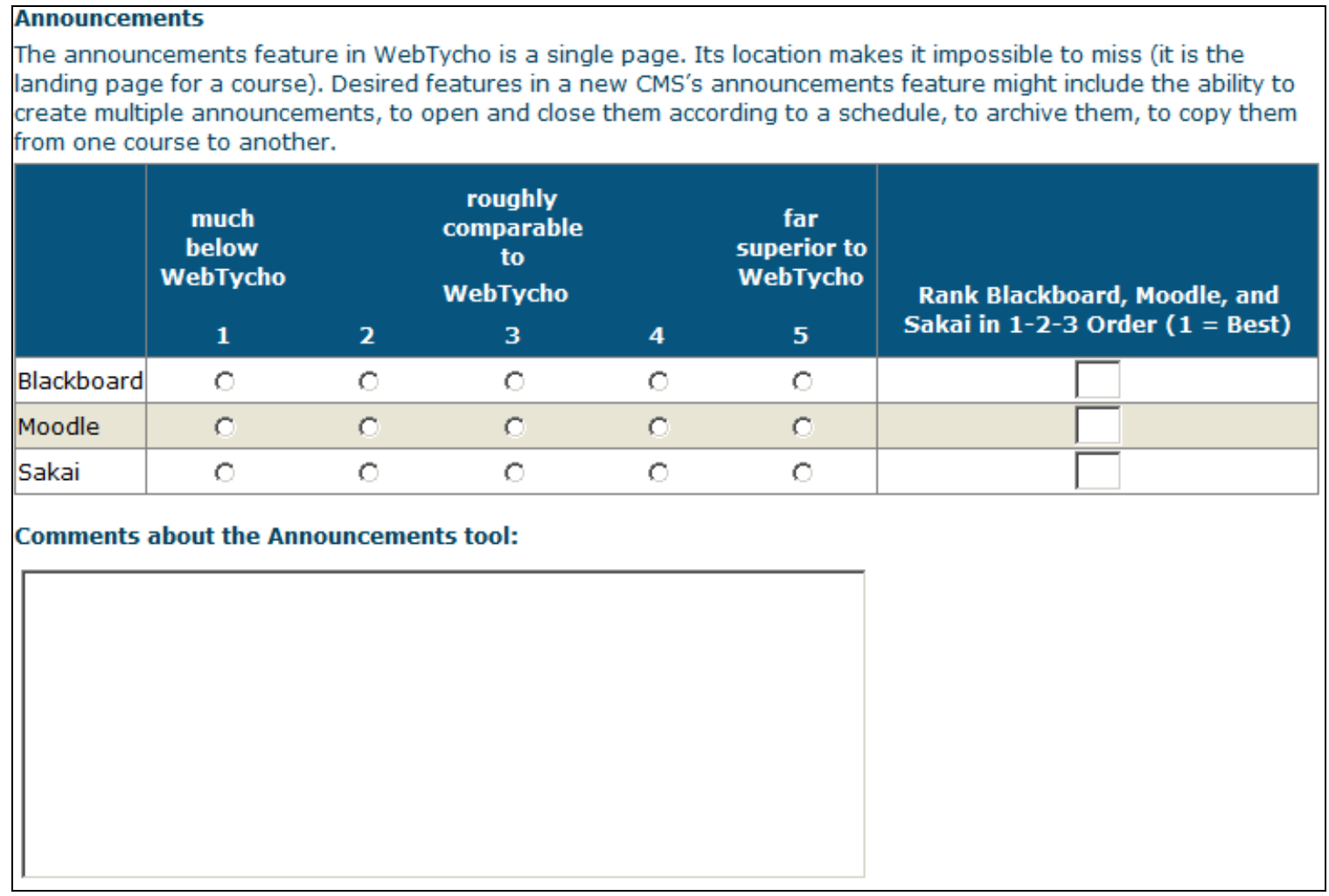

Figure 1: Rank and Rate Rubric for Selected Systems 


\section{Selection and Modification}

\section{Select a System}

Based on responses and discussion, while there was a clear preference for Sakai, any of the three CMS systems under consideration (Blackboard, Moodle, and Sakai) would be acceptable to stakeholders in terms of functionality, and each was judged to be superior to the current system, so none of the three was ruled out at this point. Sahay and Gupta (2003) provide a list of information technology related issues in supply chain management. We have modified and customized them to apply to our environment. Though there was no "formal" evaluation, the following items were important in initial selection.

\section{Is it economically feasible?}

- fixed costs

- maintenance expenses

- initial software cost

Budget was an issue, and it was necessary to look at start-up as well as on-going costs necessary for CMS maintenance. Moreover, there was the question whether the CMS should be hosted at UB or whether the hosting should be outsourced.

\section{Hosting vs. outsourcing}

For each CMS, estimates were prepared for the costs of both alternatives: hosting by UB and outsourcing. In each case, it was determined that self-hosting would be more costly both initially and over time. UB would have to acquire new hardware and new technicians to manage the CMS. To consider one example of what would be involved, it is important to have back-up equipment for a CMS because any server might fail or become overloaded. While a CMS hosting company could leverage its economies of scale and distribute UB instances across existing servers that are used by other client institutions and could easily add new servers as needed, UB would have to maintain its own set of redundancies. Moreover, since UB has never hosted a CMS in its decade of online education, there would have to be changes in the institutional culture as well within the Office of Technology Services (OTS) department. While UB's OTS commonly uses Friday evenings as a window for upgrades and patches, that same period is prime time for faculty and students using our CMS.

\section{Proprietary vs. open source system}

Since UB has a wide variety of stakeholders (novice to experts), it was necessary to have a CMS that is agile and can adapt easily to changing requirements. Our experience with proprietary systems in the past has made us wary that another such system might well be less flexible than desirable: that there would be more pressure on UB to conform to the limitations of the CMS. While the mere fact that a system is open provides no guarantee of flexibility, both of the two opensource CMSs under consideration have large and energetic client bases and a history of innovations generated at the institutional level that were subsequently made available to the broader community of Moodle or Sakai users.

\section{Flexibility to fine-tune the system}

UB is growing, and an increasing number of courses are being offered online. Flexibility was therefore a key consideration for any system that was to be selected. An open source system pro- 
vides much more freedom since source code is available and can be fine-tuned to meet client's requirements. This also eliminated proprietary CMSs.

\section{Vendor reputation}

UB has gone through four different CMS systems due to mergers or vendor problems. It was necessary to select a system where vendors would be reliable and not be likely to go out of business. This factor should favor a dominant proprietary system; however, open-source systems where there is a large, well-developed, cooperative, and growing institutional user base as well as multiple companies providing hosting can also be sufficiently reliable. Moreover, since the CMS itself is free it can be tweaked to individual requirements, and should a hosting company prove unsatisfactory, UB could switch to a competitor that hosts the same CMS or revisit the option of hosting in-house.

UB's analysis of the economic feasibility issues further strengthened the preference for Sakai. It may be useful at this point to repeat that our purpose here is to outline our procedures, not to recommend one CMS over another as the best for all situations. A different institution might well come to a different choice--for example, a larger institution with a successful history of hosting its own CMS instance might prefer to do its own hosting. Based on the above considerations and rubrics, Sakai was selected as the CMS system for UB.

\section{Operationalize the System}

Once the system was selected the next step was to decide on its operationalization. The obvious question was whether to host it internally or outsource it. It was decided to outsource the system since UB did not want to invest in $24 \times 7$ support and hire additional personnel or commit to internal resources. RFPs were collected for hosting and support. A vendor was selected based on its reputation and previous experience with hosting and $24 \times 7$ support. This CMS platform was put in place in early 2011 for classes starting in summer 2011.

\section{Modify to Meet Stakeholder's Requirements}

As mentioned above, UB hired an outsourcing company to host the Sakai instance to make some customization and modifications to the new system and to help UB integrate Sakai with other existing UB systems. With our previous CMSs, UB always had a 24/7 dedicated telephone and online helpdesk; with Sakai, the level-1 helpdesk has been outsourced to a company that has expertise in Sakai support.

\section{Implementation}

\section{Train Stakeholders}

Before the system can be implemented university wide, it is necessary to have personnel that are experts in the software. Two current WebTycho experts were trained in Sakai, both as local administrators and as trainers of ultimate users, and a plan was developed to introduce Sakai in a phased manner (Haag \& Cummins, 2010). Training classes were held for early adopters of the system. Training is ongoing and will continue until all stakeholders are trained.

\section{Make It Fully Operational}

The e-Learning Center worked closely with OTS to make sure that Sakai would be more closely integrated with existing UB systems than our previous CMS had been. PeopleSoft programmers developed extracts that could be used by Sakai for the creation of user accounts and course shells. The extracts are created every four hours, assuring that additions and deletions in the official UB 
database are quickly reflected in Sakai. The OTS network engineers worked with our Sakai hosting company to deploy Shibboleth for user authentication to ensure that students and faculty would use the same usernames and passwords in Sakai that they had for UB network access and email.

The system is currently fully operational and more and more stakeholders are being trained. A 24/7 help line is provided to assist users. The next challenge will be to measure its success. Preliminary reaction from students and faculty has been generally favorable, and calls to the Sakai helpdesk for this new CMS have typically run only slightly above the numbers that were seen with WebTycho after it had already become well established. Like any system, Sakai will evolve over time as more stakeholders use it and their needs change.

\section{Pitfalls and Lessons Learned}

As we finish this project, we have learned some interesting lessons in selecting the CMS system, which are described in the paper. However, we also learned some things that should be avoided. We have divided them as lessons learned for procurement and lessons learned for implementation.

\section{Lessons Learned for Procurement}

- Develop a task force of all users who have stakes in the system's success, otherwise some user needs and requirements may not be addressed.

- Clearly identify critical CMS features as required by all users. These may vary from university to university and with use of the system. An incomplete list can result in a suboptimal system.

- Make sure to assess each of the features/capabilities in all the platforms. Compare feature by feature in each of the selected software--otherwise it is possible to miss some critical features.

- Make the comparison as robust as possible to avoid future problems.

- Have open meetings to discuss and compare selected systems.

- Vendor reputation and longevity is important as CMS vendors are experiencing frequent changes due to merger and consolidations.

- Purchase systems that are scalable for future expansions.

- If selecting an open source system, stay engaged in the open software community for updates and patches since there may not be formal notifications.

- Do not buy a system just because everyone else is using it. Make sure it meets your specific needs to encourage maximum usage.

- Have an open system which can be seamlessly transferred to another system in case the selected system does not work as well as had been hoped.

\section{Lessons Learned for Implementation}

- Plan on a phased implementation to avoid massive training of users. The system should be implemented during a slow usage period to allow for smooth transition. The timing of the procurement process should be coordinated with the timing of the expected deployment. The optimum roll-out time for a new CMS at UB is the summer term. Counting backwards from the targeted opening date will suggest the timing that is appropriate for the earlier work of the task force. 
- Have continuous phased-in training to avoid disappointments and frustrations.

- Adjust later training sessions based on the additional feedback learned from the experiences of early users.

\section{Pitfalls to Avoid}

\section{Inertia}

Converting from one CMS to another is an enormous task and is not one to be undertaken lightly, but it is certainly achievable. If there is considerable dissatisfaction with the current CMS, it may well be worth exploring a change. UB is now on its fourth CMS in twelve years. The stakeholders continue to be progressively satisfied with each new CMS after an initial adjustment period.

\section{Imposition from above}

It is recommended there should not be any unwarranted executive interference in decisionmaking for CMS selection, especially when financials are not as important as the CMS's functionality. UB avoided this pitfall in the procurement of the new CMS. While the university did not offer any administrative support to the CMS task force carte blanche in its investigation of replacement platforms for WebTycho, it was made clear from the start that greater weight would be given to a CMS's acceptability and performance in the features that are important to its primary users than to the financial requirements. In this case, if financial or other considerations had caused the administration to select one of those other two, that result might have disappointed the task force to the extent that their top choice among three excellent alternatives was not chosen.

\section{First impressions}

When students and faculty who are used to one CMS are put into another, the first thing they will notice is that it is different, and the very fact of the difference may bias people against the new system. It is important that those evaluating a CMS spend enough time using it to become comfortable with its layout and features. Students taking an online course may use the CMS hundreds of times during a semester, and the fact that any new system will initially seem strange should not in itself be a reason for rejecting it.

\section{Current Status}

As compared to earlier CMSs used at UB, the academic community is looking forward to many additional desired CMS features for effective and enhanced teaching and learning environment. Based on our experience with the selection process, we list a few of these additional features of the proposed CMS in Table 4: 
Table 4: Additional features of the proposed CMS

\begin{tabular}{|l|l|}
\hline \multicolumn{1}{|c|}{ Feature } & \multicolumn{1}{|c|}{ Details } \\
\hline Alert Announcements & $\begin{array}{l}\text { Automatic notification of changes in the course or the } \\
\text { submission of new conference posts. }\end{array}$ \\
\hline File Management & $\begin{array}{l}\text { The ability to upload zip files and to create folders or } \\
\text { subdirectories within the file area. }\end{array}$ \\
\hline Improved Assignments Downloading & $\begin{array}{l}\text { Batch downloading/uploading of assignments for grading } \\
\text { - downloading a set of assignments in a single zipped file. }\end{array}$ \\
\hline Unique Roles and Permissions & $\begin{array}{l}\text { The ability to view the course from other perspectives-- } \\
\text { faculty, student, evaluator, guest, etc. }\end{array}$ \\
\hline Consistent Course View & $\begin{array}{l}\text { The ability to design permissions and options for various } \\
\text { aspects, for example, to add, edit, or delete entries. }\end{array}$ \\
\hline Navigation Facilitation & $\begin{array}{l}\text { A more uniform course view from one role to another, } \\
\text { except for changes in the options for permissions. }\end{array}$ \\
\hline Course Creation & $\begin{array}{l}\text { Ease of navigation from one screen to another the CMS } \\
\text { remembers the last visited screen. }\end{array}$ \\
\hline Grade Management & $\begin{array}{l}\text { The ability to import various tools, such as math tem- } \\
\text { plates. }\end{array}$ \\
\hline E-mail Server & $\begin{array}{l}\text { Ease of importing scores back and forth from Excel into } \\
\text { the gradebook. }\end{array}$ \\
\hline $\begin{array}{l}\text { E-mails supported by its own internal CMS server instead } \\
\text { of users' personal e-mail servers such as Google, Hot- } \\
\text { mail, or Yahoo. }\end{array}$ \\
\hline and
\end{tabular}

\section{Conclusion}

Course management systems are continually evolving and will keep adding capabilities and functionalities. They will gradually become more potent, not only for e-learning but also for the etraining of practitioners. In this paper, we provided one case study where one university developed a survey using desirable criteria discussed in Adlakha and Aggarwal (2009) for the selection process of a new improved CMS to replace the existing CMS. In addition, the paper discusses pitfalls that should be avoided in selecting a CMS. Universities planning to venture into elearning could learn from our experiences before selecting a CMS tool. This case study presents a hybrid methodology for procurement; however, each institution will have to modify it to tailor it to their needs.

\section{References}

Adlakha, V. G., \& Aggarwal, A. K. (2009). Minimal functionalities of course management systems: A faculty perspective. International Journal of Web-Based Learning and Teaching Technologies, 4, 27-43.

Aggarwal, A. K., \& Makonnen, P. (2008). Critical success factors (CSF) for successful globalized elearning. International Journal of Innovation and Learning, 6, 92-109. 
Al-Mahmood, S., \& Mansoor, A. A. (2011). COTS software procurement methodology. In Eishaa Alikhaliffa (Ed.), E-strategies for resource management systems (pp. 288-306). IGI Global.

Babou. (2008). What is stakeholder analysis? - Part 3. Retrieved January 17, 2011, from http://leadershipchamps.wordpress.com/category/stakeholder-management

Burd, S., Seazzu, F., \& Conway, C. (2009). Virtual computing laboratories: A case study with comparisons to physical computing laboratories. Journal of Information Technology Education: Innovations in Practice, 8, 55-78. Retrieved from http://www.jite.org/documents/Vol8/JITEv8IIP055078Burd693.pdf

Christensen, C. (2008). Disruptive innovation in higher education. Forum Futures. Retrieved from http://net.educause.edu/ir/library/pdf/ff0810s.pdf

Christensen, C., \& Horn, M. (2008). How do we transform our schools? Education Next, 8, 13-19.

CMS Task Force Survey. (2010). Retrieved July 13, 3012, from http://webteach.ubalt.edu/UltimateSurvey/Surveys/TakeSurvey.aspx?s=13F0E7E96555462D9D1DB9 8179BD63F9\&responseGuid=9427b091-775f-4c86-80de-91e84997c5a6

Dillenbourg, P. (2000). Virtual learning environments. Workshop at EUN Conference 2000: Learning in the new millennium: Building new education strategies for schools. Accessed on June 12, 2012, from http://tecfa.unige.ch/tecfa/publicat/dil-papers-2/Dil.7.5.18.pdf

Haag, S., \& Cummins, M. (2010). Management information systems for the information age. McGraw-Hill.

Kerzner, H. (2009). Project management case studies (3rd ed.). Wiley.

Laudon, K. C., \& Laudon, J. P. (2010). Management information systems: Managing the digital firm (11th ed.). Prentice-Hall.

Picciano, A., Seaman, J., \& Allen, I. E. (2010). Educational transformation through online learning: To be. Journal of Asynchronous Learning Network (JALN), 14(4), 17-35.

Sahay, B. S., \& Gupta, A. K. (2003). Development of software selection criteria for supply chain solutions. Industrial Management \& Data Systems, 103(2), 97-110.

Sangrà, A., \& González-Sanmamed, M. (2011). The role of information and communication technologies in improving teaching and learning processes in primary and secondary schools. Journal of Asynchronous Learning Network (JALN), 15(4), 207-220.

Santala, R., \& Samuli, S. (2010). Spend management: Key elements for realising cost savings in procurement. Bearing Consulting group. Retrieved on May 23, 2012, from http://www.bearingpoint.com/enother/download/Spend_Mgmt WP FI 8p.pdf

Sener, J. (2010). Why online education will attain full scale. Journal of Asynchronous Learning Network (JALN), 14(4), 3-16.

Strong, K. C., Ringer, R. C., \& Taylor, S. A. (2001). THE* rules of stakeholder satisfaction (*Timeliness, honesty, empathy). Journal of Business Ethics, 32(3), 219-230.

Unal, Z., \& Unal, A. (2011). Evaluating and comparing the usability of web-based course management systems. Journal of Information Technology Education, 10, 19-38. Retrieved from http://www.jite.org/documents/Vol10/JITEv10p019-038Unal904.pdf

Walter, E. (2010, April 7). Disruptive innovation theory and public school education: A futurist perspective [Web log post]. Retrieved July 22, 2010, from http://urbned.wordpress.com/2010/04/09/disruptiveinnovation-theory-and-public-school-education-a-futurist-perspective/ 

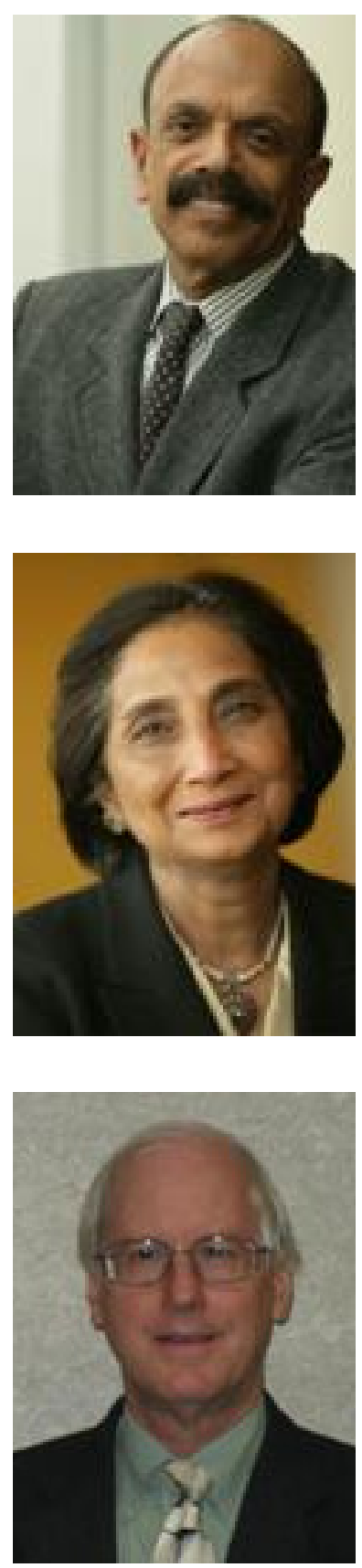

\section{Biographies}

Dr. Anil Aggarwal is a professor in the Merrick School of Business at the University of Baltimore. Dr. Aggarwal has published in many journals, including Computers and Operations Research, Decision Sciences, Information and Management, Production and Operation Management, Total Quality Management \& Business Excellence, eService, Journal of EUC and many national and international professional proceedings. He has edited two books on web-based education and is currently editing a book on cloud computing. His current research interests include Web-based education, Global Information Systems, Virtual Teams and cloud computing.

Dr. Veena Adlakha is a professor of Production Management at the Merrick School of Business at the University of Baltimore. Her research on discrete event simulation, stochastic activity networks, and fixed-charge transportation problem, web-based education has appeared in several journals including Networks, European Journal of Operational Research, Management Science, Operations Research, OMEGA, JORS, Total Quality Management \& Business Excellence, and e-Service Journal. Her current research interests include fixedcharge transportation problem, total quality management and webbased education.

Terry Ross is the Program Manager for e-Learning at the University of Baltimore. His research interests include innovations in online education and computer-assisted applications in literary authorship studies. 